\title{
Successful Endovascular Management of Post-Traumatic Phlegmasia Cerulea Dolens from Rupture of the External Iliac Vein
}

\author{
Chan Yong Park, M.D., Ph.D., Hyun Min Cho, M.D., Ph.D., \\ Kwang Hee Yeo, M.D., Ph.D., June Pill Seok, M.D., Chan Kyu Lee, M.D. \\ Department of Trauma Surgery, Pusan National University Hospital, Busan, Korea
}

Correspondence to:

Chan Kyu Lee, M.D.

Department of Trauma Surgery,

Pusan National University

Hospital, 179 Gudeok-ro,

Seo-gu, Busan 49241, Korea

Tel: +82-51-240-7369

Fax: +82-51-240-7719

E-mail: apolo1980@hanmail.net
We report a rare case of a 47-year-old male with posttraumatic phlegmasia cerulea dolens caused by a ruptured right external iliac vein and treated with an endovascular venous stent graft. The patient was the victim of motor vehicle accident, and suffered direct injuries to the head and abdomen. The patient had a cyanotic and swollen right lower leg. Abdominal and lower extremity computed tomography angiography revealed a large retroperitoneal hematoma caused by a ruptured right external iliac vein, and grade I liver injury. The right external iliac vein rupture was successfully treated with a venous stent graft, followed by inferior vena cava filtering, because a venous thrombus was identified below the stent graft. He initially was hemodynamically unstable but recovered following treatment. The patient was comatose when presenting at the emergency department. He was discharged, fully recovered, on hospital day 18. (J Acute Care Surg 2017;7:87-89)

Key Words: Posttraumatic phlegmasia cerulea dolens, Ruptured external iliac vein, Endovascular venous stent graft, Inferior vena cava filtering, Venous thrombus

Received July 6, 2017, Revised September 18, 2017, Accepted September 20, 2017

Copyright $\odot 2017$ by Korean Society of Acute Care Surgery

(c) This is an Open Access article distributed under the terms of the Creative Commons Attribution Non-Commercial License (http://creativecommons.org/licenses/by-nc/4.0) which permits unrestricted non-commercial use, distribution, and reproduction in any medium, provided the original work is properly cited.

\section{Introduction}

Phlegmasia cerulea dolens (PCD) is a rare form of massive proximal (e.g., iliofemoral) venous thrombosis of the lower extremities associated with a high degree of morbidity, presenting with sudden severe lower extremity pain with edema and cyanosis [1]. Because of its rarity, there have not been well established treatments. Several reports have described about surgical treatment, but only a few reports has been reported for interventional treatment. In our case, the patient had suffered by PCD caused by external iliac vein (EIV) rupture. We initially considered surgery, but we performed interventional venous stent graft because the patient was hemodynamic unstable and not suitable for surgery.

\section{Case Report}

A 47-year-old male was transferred to the emergency department after pedestrian traffic accident on a street. He was hemodynamically unstable, with blood pressure $50 / 30 \mathrm{mmHg}$, heart rate 113 beats/min, and respiration 18 times/min. He was initially drowsy with Glasgow Coma Scale of 12 at the emergency 


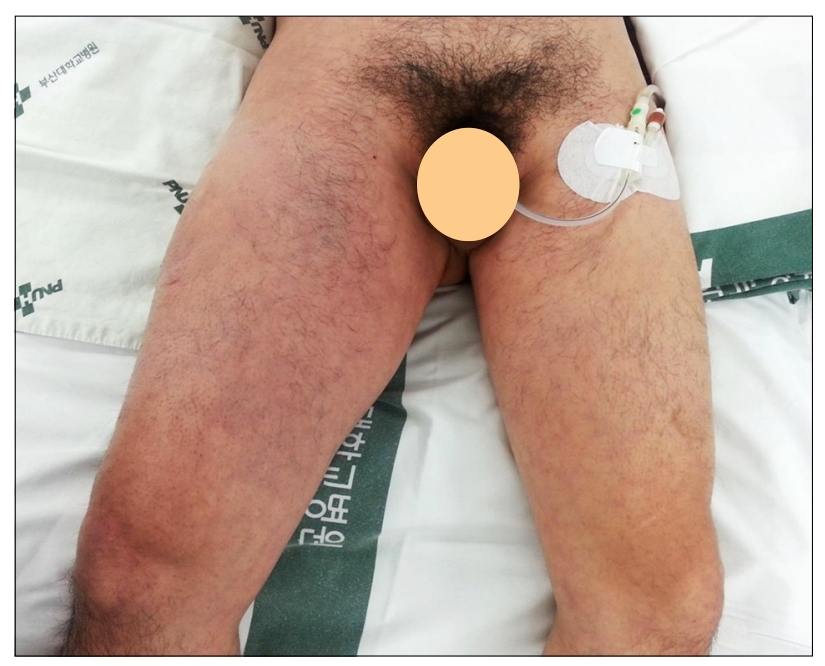

Fig. 1. Swelling and cyanotic color change of the right thigh were found at initial presentation.

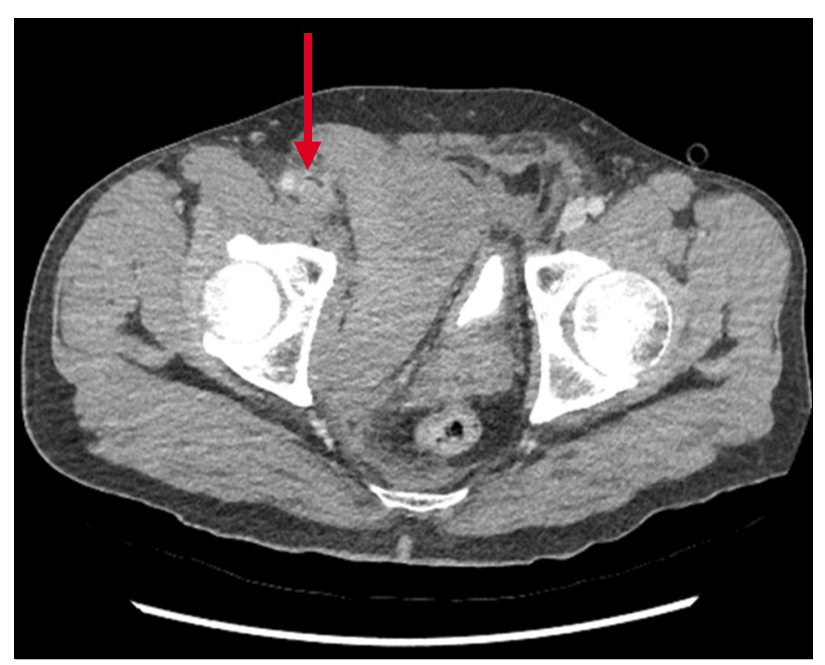

Fig. 2. Computed tomography angiography showed the extravasation (arrow) from the right external iliac vein.

department. He was intubated on arrival and received vigorous fluid resuscitation. On physical examination, his right leg was massively swollen, cyanotic and cool (Fig. 1). Brain computed tomography (CT) revealed traumatic subdural hematoma, epidural hematoma and multiple hemorrhagic brain contusion with the skull fractures. Abdominal and lower extremities CT angiography revealed large retroperitoneal hematoma caused by ruptured right EIV and grade I liver injury. Both lower extremities' arterial flows were intact (Fig. 2).

The patient was emergently transferred to the intervention unit
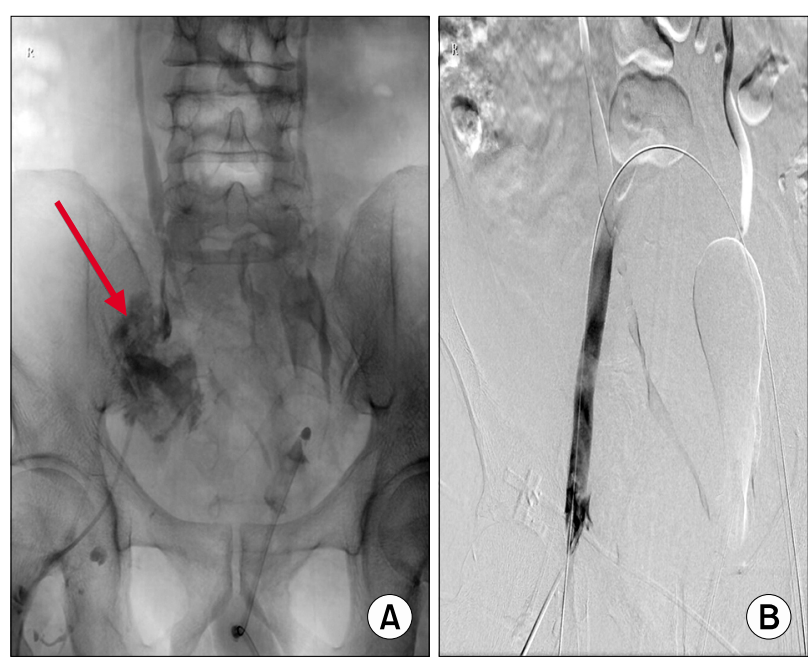

Fig. 3. (A) The arrow indicates extravasation from the right external iliac vein. (B) It shows no further extravasation by successful venous stent grafting.

for endovascular repair. Through the right common femoral vein (CFV) access, venography was performed and it revealed contrast extravasation through the right EIV. Guide wire was advanced into the inferior vena cava (IVC) from the CFV. Then, the Viabahn endoprosthesis (Gore \& Associates, Flagstaff, AZ, USA) were deployed in an overlapping configuration spanning the right EIV. The grafts were then expanded with balloon dilatation catheter, and extravasation was terminated. Subsequent venography was performed through the right $\mathrm{CFV}$ access and it revealed restored patency of the EIV (Fig. 3). Right after the stent grafting, the patient gained hemodynamic recovery and the color and temperature of the right leg were also improved. However, partial thrombotic obstruction was identified below the venous stent. Because of the inability to use anticoagulants due to the patient's brain injuries, IVC filter was inserted to prevent further pulmonary thromboembolism.

The patient was transferred to the intensive care unit after achieving recovery from hypovolemic shock and was discharged the hospital on hospital day 18. There was no complication during the follow-up period up to 6 months.

\section{Discussion}

PCD is a rare condition caused by complete venous occlusion 
that leads to impaired arterial flow, especially PCD resulting from trauma has been reported only a few [2-4]. Because of rarity of PCD, the treatment options have not been well documented. Several cases have been reported of blunt iliac vein injury without a pelvic fracture, and surgical repair has traditionally been the method of treatment $[5,6]$. With other treatment, Steven R. et al. have reported successful treatment of a patient with traumatic iliac vein rupture who was not a reasonable surgical candidate by using endovascular stent graft [7]. In our case, the patient was initially hemodynamically unstable but was partially recovered with early resuscitation. We considered vascular surgery and angiographic intervention as a treatment for the ruptured EIV, but we thought the patient was not suitable for surgical candidate. Generally, vascular surgery needs the use of anticoagulants such as heparin. The patient had liver laceration and cerebral hemorrhage, so we was worried about aggravation of internal bleeding after vascular surgery. We thought intervention was safer than open vascular surgery which needed bypass graft and heparin injection.

In the case of a patient who has uncontrolled multiple bleeding focuses, endovascular stenting could be a good alternative as the treatment of the EIV injury instead of vascular surgery.

\section{Acknowledgments}

This work was supported by clinical research grant from Pusan
National University Hospital in 2017.

\section{Conflicts of Interest}

No potential conflict of interest relevant to this article was reported.

\section{References}

1. Vysetti S, Shinde S, Chaudhry S, Subramoney K. Phlegmasia cerulea dolens: a rare, life-threatening condition. Scientific World Journal 2009;9:1105-6.

2. Chinsakchai K, Ten Duis K, Moll FL, de Borst GJ. Trends in management of phlegmasia cerulea dolens. Vasc Endovascular Surg 2011;45:5-14.

3. Stallworth JM, Bradham GB, Kletke RR, Price RG Jr. Phlegmasia cerulea dolens: a 10-year review. Ann Surg 1965; 161:802-11.

4. Wilson B, Hawkins ML, Mansberger AR Jr. Posttraumatic phlegmasia cerulea dolens: an indication for the Greenfield filter. South Med J 1989;82:780-2.

5. Bracale G, Porcellini M, D'Armiento FP, Baldassarre M. Spontaneous rupture of the iliac vein. J Cardiovasc Surg (Torino) 1999;40:871-5.

6. Buice WS, Hollenbeck JI, McElwee T. Isolated iliac vein injury from blunt trauma. Surgery 1990;107:350-2.

7. Zieber SR, Mustert BR, Knox MF, Fedeson BC. Endovascular repair of spontaneous or traumatic iliac vein rupture. $\mathrm{J}$ Vasc Interv Radiol 2004;15:853-6. 